\title{
Research on FE Modeling Common Method and Integration System for Nuclear Power Valve
}

\author{
DAI Ye ${ }^{1}$, LI Yuan ${ }^{2 *}$, WANG Jian ${ }^{3}$ and LI Da-meng ${ }^{1}$ \\ ${ }^{1}$ School of Mechanical \& Power Engineering, Harbin University of Science and \\ Technology, Heilongjiang Harbin 150080, China \\ ${ }^{2}$ School of Mechanical Engineering and Automation, University of Science and \\ Technology LiaoNing, Liaoning Anshan 114051,China \\ ${ }^{3}$ Department of Mechanical and electrical Engineering, East University of \\ Heilongjiang, Harbin, China. 150086, China \\ *liyuanlnkj@163.com
}

\begin{abstract}
Because of the high security, shape complexity of valve cavity and the nonlinear of joint surface at sealing position, establishing the finite element model quickly and accurately that could meet engineering accuracy and calculation condition is the problem that need urgently solved. A new method on knowledge reuse process of finite element analysis among valve similar model is presented based on modular thought, which could effectively improve the simulation accuracy and efficiency. Evaluation index system would be constructed according to the characteristics of nuclear power valves in order to perfect the technical standard and the norm of nuclear power valve design in our country. And on these bases above, the integrated platform as practical engineering tools could be constructed, which integrates design, simulation, and optimization of nuclear power valves together supporting heterogeneous model transfer. It is expected to give theoretical development and practical application to improve the design ability and the product performance of nuclear power valves.
\end{abstract}

Keywords: nuclear power valve, reuse process, collaborative design, system design

\section{Introduction}

Among the power plant equipments, power station valves as the key components control and regulate the flow and pressure of the medium inside the tubes, to ensure power plant equipments normal and safe working, that are important guarantee for the entire power plant to stabilize operation. To improve the designing quality and shorten the research and development cycle of power station valves has become the key of the enterprises to enhance their competitiveness [1-3]. Therefore, developing platform with rapid design of three-dimensional model, multi-disciplinary finite element characterization and multi-disciplinary optimization design for power station valves has important theoretical significance and practical value.

As the progress of nuclear power industry in our country, valve products of the nuclear power station are in the period of rapid development, and the requirements of high performance, low cost and short cycle are put forward to the design of nuclear power valves, hence advanced digital design methods and integrated design system provide technical support in urgent need. Especially under leakage of nuclear power station in Japan, there is important realistic significance to improve design ability of nuclear power valves and product performance. The most of nuclear power valve design at home is given priority to with deformation design, namely, according to the customer's individuation demand with reference to the original mature products, a new type of 
nuclear power valve products is usually designed by the retrofit design on the basis of the introduced mature technology [4-6].

However, there exist a universal phenomenon of low efficiency and high processing cost during the valve manufacture process in domestic nuclear power valve business. In addition, there is no reasonable design process model as guidance in the process of constructing the design flow of integrated system of the nuclear power valve products $[7,8]$. Although same enterprises have introduced the technologies of computer aided design (CAD) and computer aided engineering (CAE), the information transmission still rely mainly on manual transmission due to the lack of interaction and mutual support between CAD and CAE. Due to the lack of the integrated support environment, this kind of scattered design method exists some shortages in the application integration of $\mathrm{CAD} / \mathrm{CAE} / \mathrm{MDO}$, it is not suitable for the current fast response design requirements of nuclear valve products. Obviously, these shortcomings greatly affect the development speed and design quality of valve products, so it is an urgent need to establish a practical CAX integrated design system. Therefore, the problem of isolated information among the CAX systems has become increasingly acute, leading to the low efficiency in the digital prototype design process, the difficulty in managing the result data and the suboptimal design performance of valve products. On the other hand, the establishment of the analysis system for nuclear power valve provides the navigation process for the MDO integrated system, and the analysis process can be converted to the analysis templates that can be used in the process of analysis reuse $[9,10]$. In this paper, the effort in the search for ways will be done to improve the nuclear power valves products by digital design technology and integrated design platform.

\section{The General Framework and Function}

\subsection{Function Analysis of the Integrated System}

The digital design system structure is constructed to satisfy the need of the performance simulation analysis of nuclear power valve products, the system structure are described herein according to level structure, it mainly consists of three major components, including the supporting layer, function layer and application layer, as illustrated in Figure 1.

According to the characteristics of the digital design process and requirements for the power station valve products, the integrated system functions of each part for collaborative design, simulation and optimization mainly are described as below:

(1) Supporting layer, which is the foundation of the whole system constructed by using current mainstream technology, is responsible for transferring the data between the functional level and the application layer in order to realize data sharing;

(2) Function layer is based on the support of software environment foundation, in which the logic modules are developed according to the business needs of the nuclear power valve products digital design, such as the parametric design logical processing based on UG, the comprehensive characteristic analysis logical processing based on ANSYS; the structure optimization design logical processing based on the ISIGHT. 


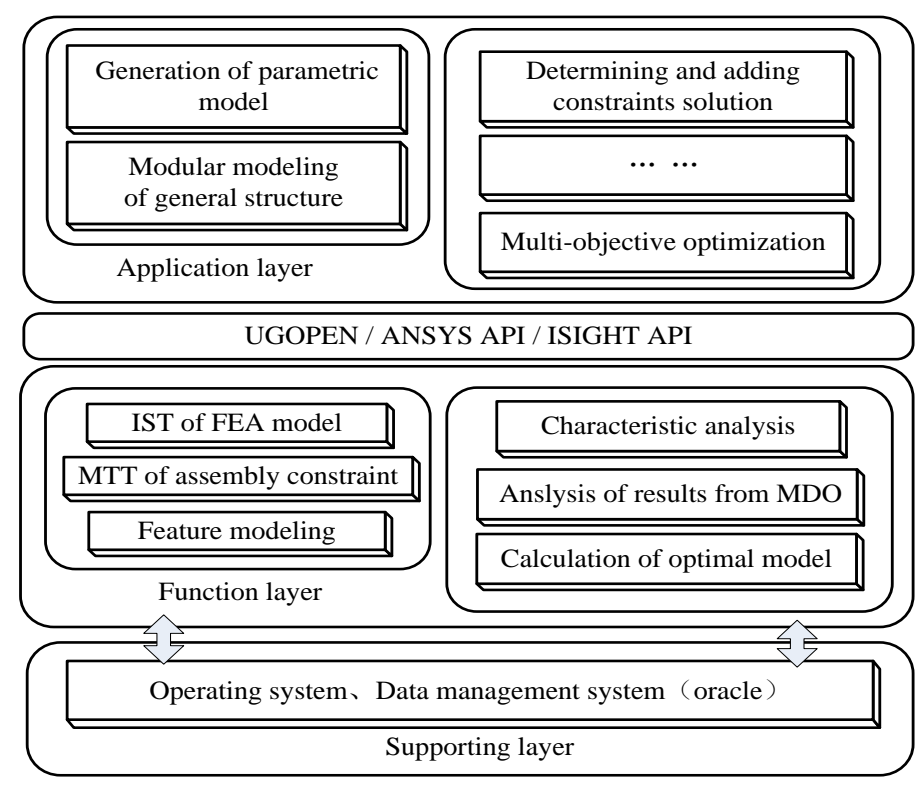

Figure 1. Digital Design Integration Platform Operation Environment

(3) Application layer, the description of usage mode in nuclear power valve design, can carry out the nuclear power valve multi-physical field analysis, which includes the valve body structure analysis, the flow properties analysis, the thermal - stress coupling analysis and the seismic analysis. The application of nuclear power valve products digital design can be integrated to modeling, characteristic analysis and optimization calculation software.

\subsection{System Construction of Multi-physical Field Analysis}

In order to construct the characteristics evaluation system of nuclear power valves, multi-physics analysis (including structural static analysis, thermalmechanical coupling analysis, flow field analysis and Seismic analysis), the whole system can adopt the initial design data of the valve product as the origin, including parametric modeling and assembly, multi-physics field analysis, structural optimization design of the nuclear power valve products.

Figure 2 illustrates the system construction of multi-physical field analysis for nuclear power valves, which is divided into four parts: mechanical analysis, fluid mechanics analysis, thermal-mechanical coupled analysis and seismic analysis. These analyses are totally based on the boundary and constrain condition of the actual working conditions so as to conform to the engineering practice. This kind of hierarchical model not only ensure the total optimization property of the entire product, but also give consideration to the independence of each subsystem so as to realize the collaboration design easily between different areas. The main steps of the integration system can be classified in four parts: (1) Parametric modeling and assembly of the valve products; (2) Valve multi-physics field analysis; (3) Valve structure optimization design: (4) Knowledge management system. 


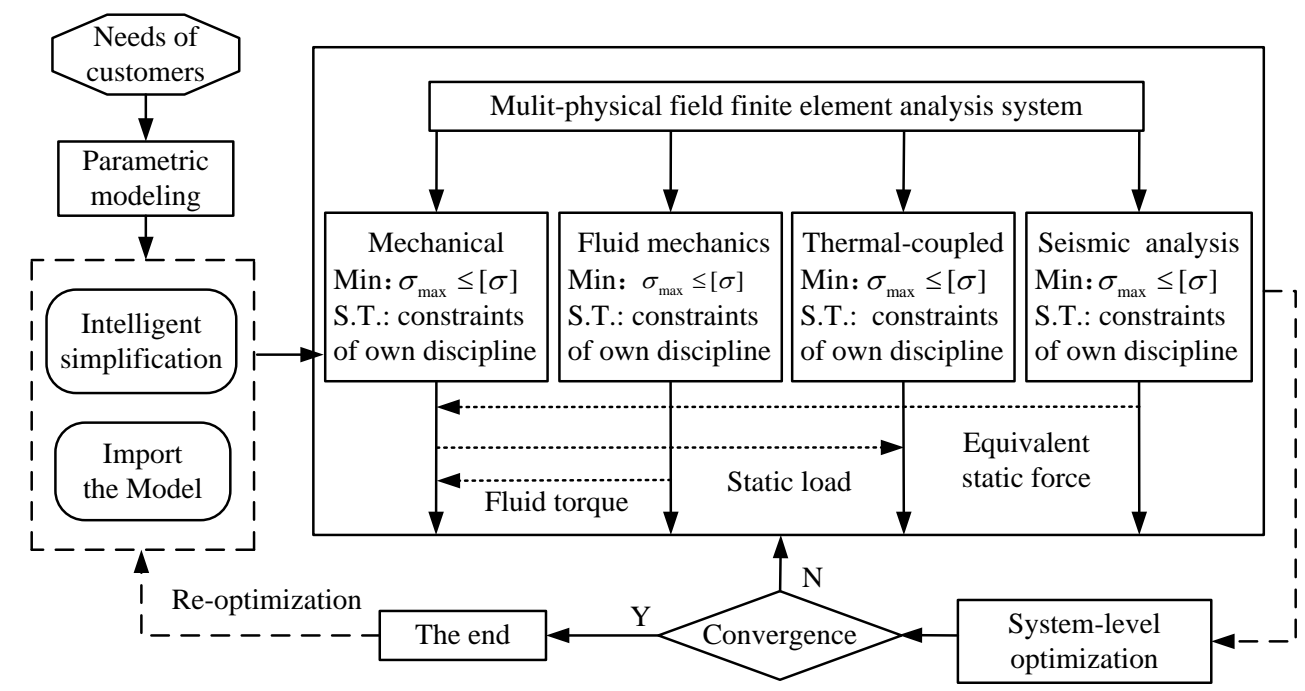

\section{Figure 2. Digital Design Flow of Nuclear Power Valve Multi-physical Field Analysis}

\section{Modeling Common Method in System Integration}

\subsection{Configuration Process of the Valve Digital Design}

The modeling method is based on the features in the process of building valves parametric models, which helps to express more specific features of model and be convenient for the operator to programme. In order to provide a visual representation of the configuration process of the nuclear power valve design system, a module connection diagram is introduced here to describe the design process expressed as shown in Figure 3.

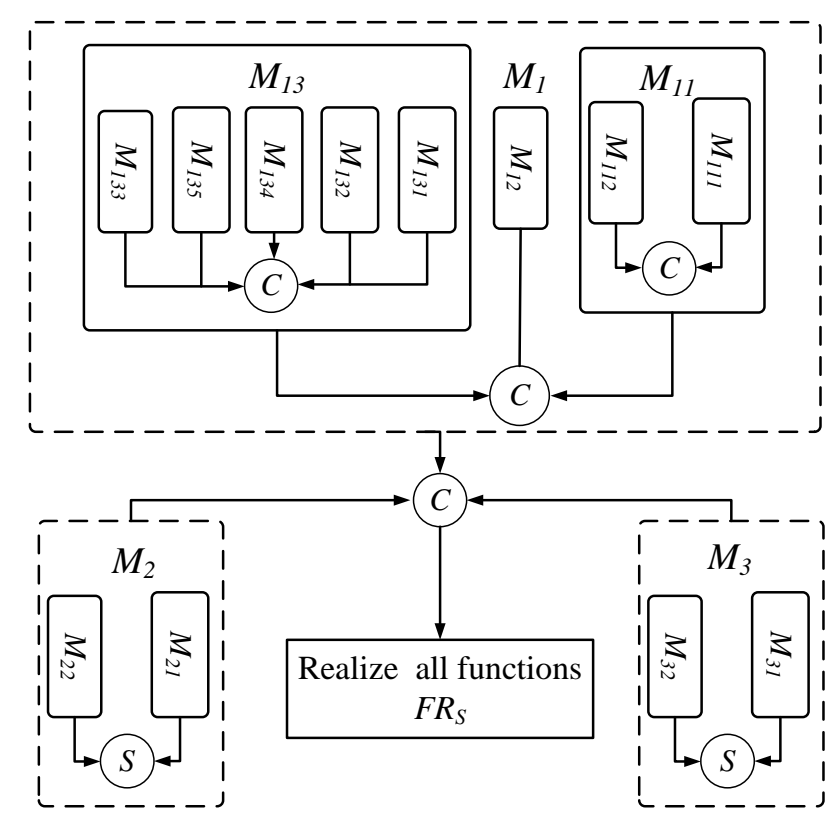

Figure 3. Valve Design Flow Chart

The symbol of " $\bigcirc$ "expresses the module joint operations so as to show the relationship among modules. For no coupling design, "circle $S$ " is used to represent the simple addition of $F R s$; "circle C" signifies that DPs and Ms must be controlled according to the decoupling order of the design matrix. The design of the valve 
design complies with the sequence from the outside to the inside, which embodies the requirement of the axiomatic design. " $M$ " in the figure stands for the design parameters. At the highest level, the nuclear power valve is composed of three sequential modules: $M_{1}$ (port opening and closing module) $--M_{3}$ (aseismatic module) $-M_{2}$ (sealing module). And $M_{1}$ can also be divided into three parts: $M_{11}$ (open-close structure module) $-M_{12}$ (support structure module) $-M_{13}$ (driving structure module). $M_{11}$ is composed of parallel module $M_{111}$ and $M_{112}$, so the design of $D P_{111}$ and $D P_{112}$ can first be determined. By the same token, the whole valve structure design order can be obtained.

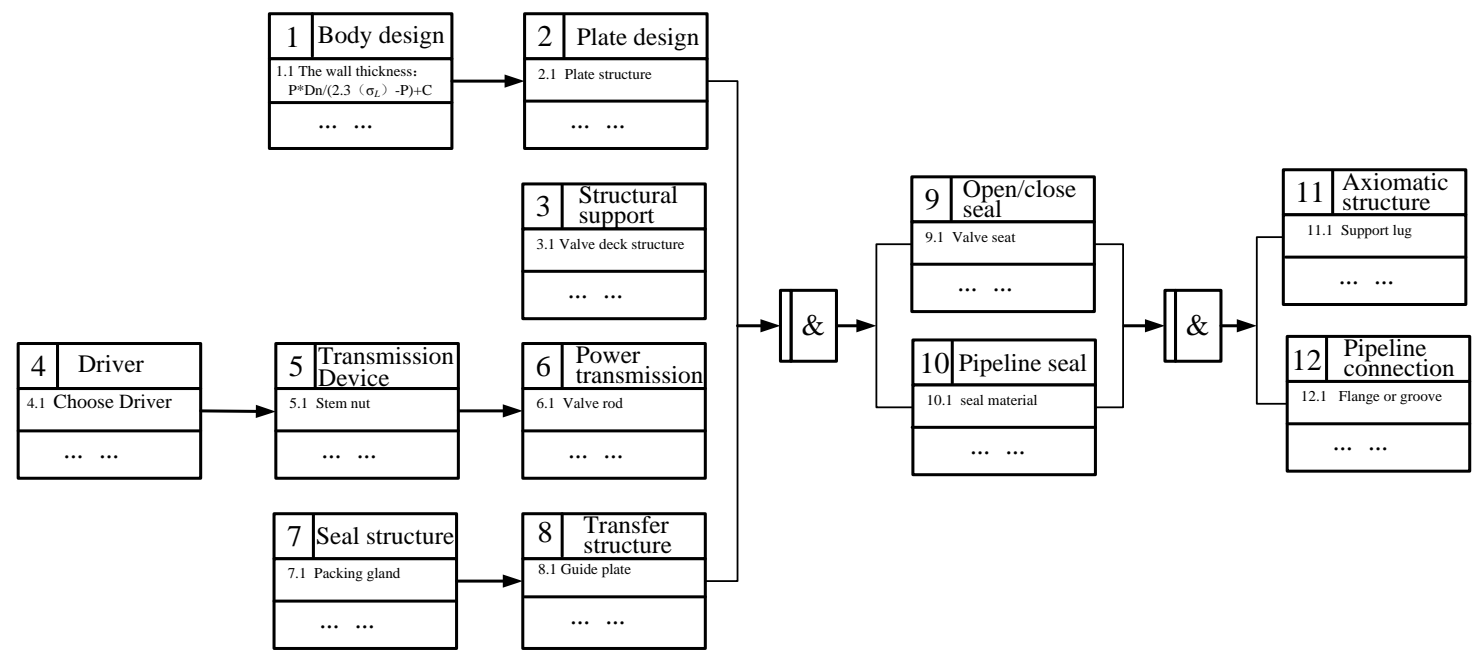

Figure 4. Management Model of Gate Valve Design Process

As shown in Figure 4, the model can describe the whole process of valve design, which has a guiding role on the digital design and management. At the same time, the design process management model for the digital design behind provides the support of the valve data structure organization.

\subsection{Reuse Process of Finite Element Analysis}

The process of generating the FEA reuse model, making the command stream processing of the typical parts standardization, contains numerical parameterization (such as characteristic parameter values, grid size, etc.,) finite element operation parameterization (such as definition of the material properties, result reduction, etc.,). The key point of reuse process as shown in Figure 5: one is to form a unified reuse data model, namely the reusable template under the XML definition; another one is the parameterized finite element modeling techniques [11]. 


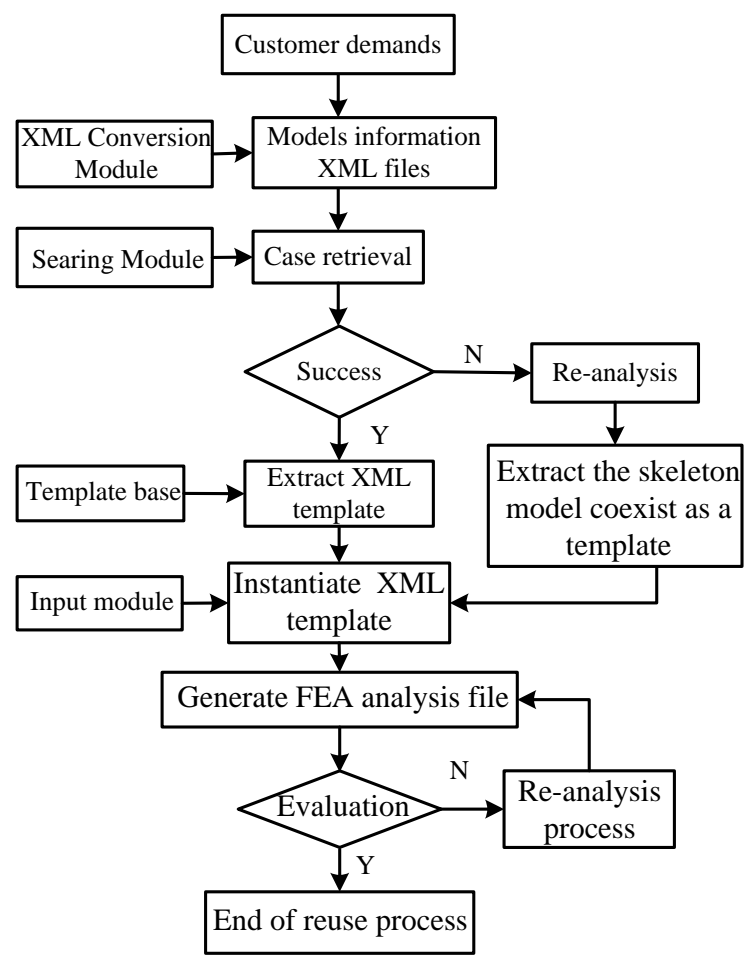

Figure 5. Rapid Analyses Reuse Process of FEA

\subsection{Multi-physics Field Analysis of the Valve}

As shown in Figure 6, in order to comprehensively analyze the specific impact of the nuclear power valve under various influence factors, multi-physics field analysis of the whole valve, including structural static analysis, thermal-mechanical coupling analysis, flow field analysis, seismic analysis, can be completed to predict the valve performance under different work conditions [12].

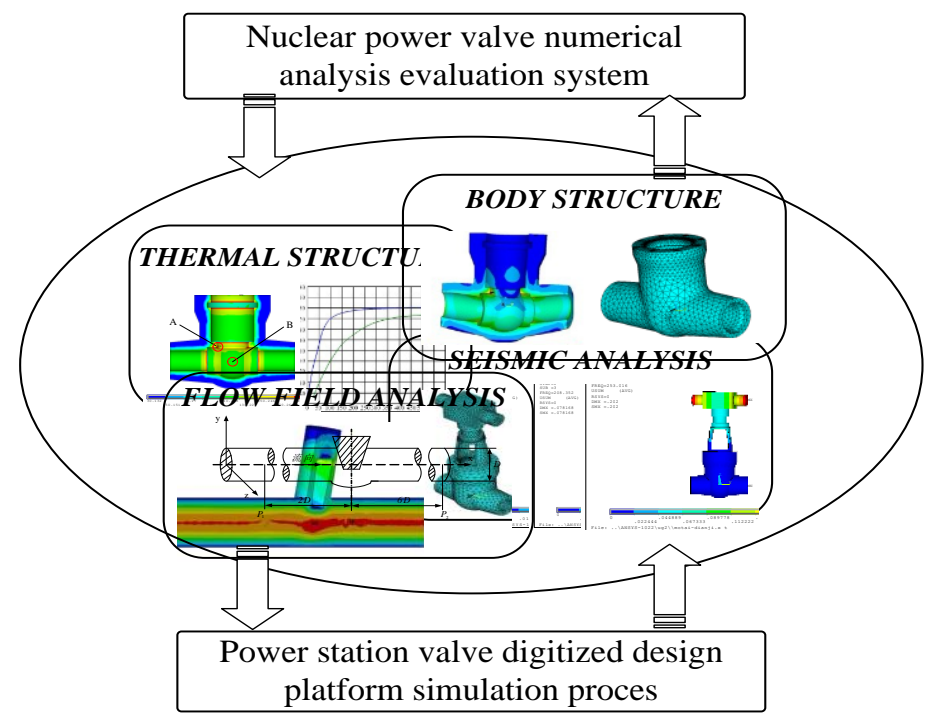

Figure 6. Multi-physics Field Analysis of the Valve

\section{Implementation and Application of the System}

The nuclear power valve integrated system for collaborative design, simulation and optimization is developed based on VC and SQL Server database system. Through 
providing a unified the data access interface, the system make each simulation system be flexible to define and publish the data sets so as to realize the information integration in the multidisciplinary simulation process.

In order to conduct multi-discipline optimization, this system integrates iSIGHT with ANSYS. When conducting the coupling operation of valve's model analysis and structural analysis, it can realize the coupling analysis by introducing command flow files(Structure.txt and Modal.txt), carrying out batch files of application, and compiling the output result files by using *Get command and /output command of command stream files. Finally, iSIGHT compares present function value with the target expected value to determine whether to iterative again.
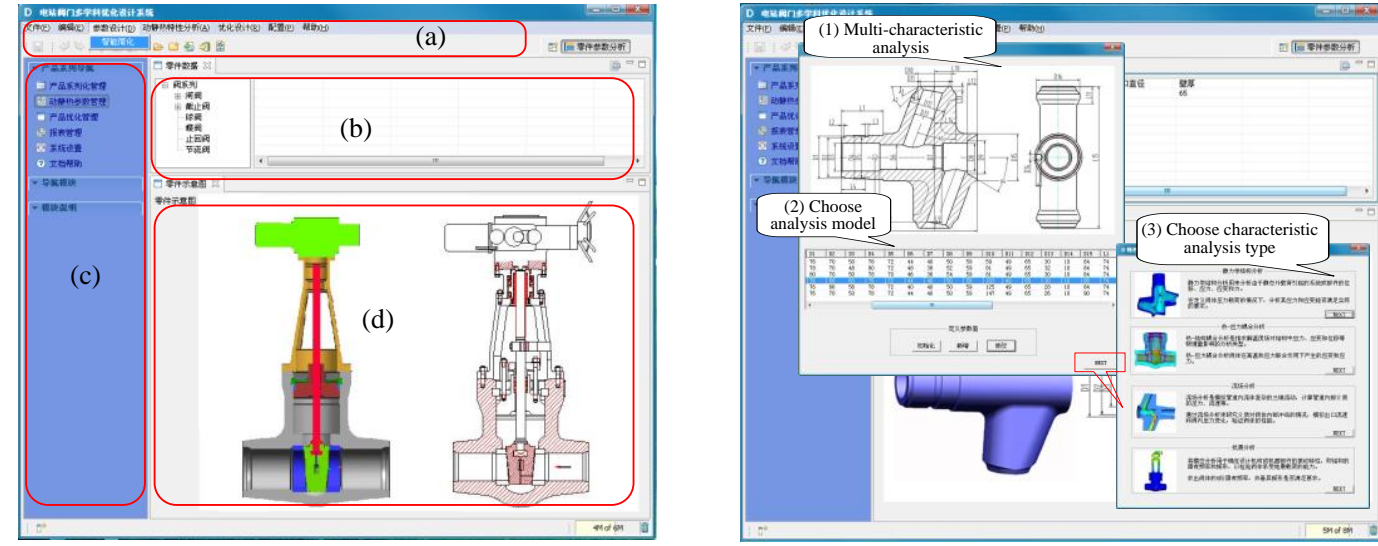

Figure 7. Main Interface of the Characteristics Analysis Process

The main interface of the integrated system for valve products is shown in Figure 7, which is divided into four functional areas, viz. (a) navigation area; (b) parts list area; (c) system management list area; (d) parts diagram area. Rapid modeling and modification of model for each component of the valves and automatic assembly of the whole machine are realized by UG secondary development. Each analysis module manages the data of the CAE model, simulation analysis and post-processing graphic. Utilization of model simplified technology to develop intelligent simplified module of model that traverses the model features automatically to simplify fine characteristics such as chamfers, small holes. The analysis results will be displayed in the form of a chart. Finally, the optimum module will optimize the structure of the valve. It increases the success rate of model transformation between CAD and FEA, and improves the analysis efficiency of CAE.

\section{Conclusion}

According to the design requirements of the power station valves in this paper, the object-oriented design ideas is used in the system based on the functional analysis and comprehensive analysis of design ideas of domestic and foreign outstanding CAX integrated platform, to put forward a framework with structure, modularity and sharing of multidisciplinary optimization design system. CAX and MDO are integrated according to the design characteristics of the power station valves. The interactive information of each module can be turned into middle format to store in a database through ADO technology, and it can be directly used for knowledge management system to ensure the information sharing and unity between modeling, characteristic analysis and optimization design. The circulation and sharing of data are solved by transferring and managing model data based on database completing the development of multidisciplinary design optimization system of the power station valves. 


\section{ACKNOWLEDGEMENTS}

The research is supported by Natural Science Foundation of Heilongjiang Province of China (Grant No. E201440), Heilongjiang Province Education Bureau Project (Grant No. 12541146), National Natural Science Foundation Project (Grant No. 51375125).

\section{References}

[1]. C. Antonio, K. G. Satyankra, D. Abhijit, et al., "Machining feature-based similarity assessment algorithms for prismatic machined parts", Computer-Aided Design, vol. 38, no. 9, (2006), pp. 954-974.

[2]. S. Yao-Jun, D. Ye and W. Nan-Qun, "Research on variant design and optimization system for nuclear power valve based on CAD/CAE integration", International Journal of Control and Automation, vol. 7, no. 9, (2014), pp. 297-304.

[3]. B. Jing, G. Shuming, T. Weihua, et al., "Design reuse oriented partial retrieval of CAD models", Computer-Aided Design, vol. 42, no. 12, (2010), pp. 1069-1084

[4]. A. M. Ji, K. Zhu, J. C. Huang, et al., "CAD/CAE Integration System of Mechanical Parts", Advanced Materials Research, no. 38, (2011), pp. 272-276.

[5]. L. Mingzhu, D. Ye and Z. Guoyin, "Research on CAD/CAE/MDO integrated system for nuclear valve products", Journal of Harbin Institute of Technology (New Series), vol. 19, no. 4, (2012), pp. 99-103.

[6]. Y. L. Perry, "Using steady flow force for unstable valve design: modeling and experiments", Department of Mechanical Engineering, no. 27, (2005), pp. 39-42.

[7]. Y. Dezhen, R. Yi, W. Zili, et al., "Design-in of reliability through axiomatic design", Journal of Beijing University of Aeronautics and Astronautics, vol. 40, no. 1, (2014), pp. 63-68.

[8]. R. Amirante, G. D. Vescovo and A. L. ippolis, A flow forces analysis of an open center hydraulic directional control valve sliding spool", Energy Convers Manage, no. 47, (2006), pp. 114 -131.

[9]. Z. Shengwen, D. Yuling, W. Guicheng, et al., "CAD/ CAPP/ CAM integration system for frame parts of marine diesel", Computer Integrated Manufacturing Systems, vol. 17, no. 4, (2011), pp. 760-766. (in Chinese)

[10].V. Naranje and S. Kumar, "An intelligent CAD system for automatic modelling of deep drawing die", International Journal of Computer Applications in Technology, vol. 48, no. 4, (2013), pp. 330-344.

[11].D. Ye, L. Yinan, R. Shengle and Y. Yuxiang, "Finite Element Analysis Process Reuse Method based on Integrated Information Model", International Journal of u- and e- Service, Science and Technology, vol. 6, no. 5, (2013), pp. 57-66.

[12].G. S. Xue, S. G. Kim and L. Wang, "Transient flow analysis of spring loaded pressure safety valve", American Society of Mechanical Engineers, vol. 7, (2010), pp. 253-258. 